\title{
Repeat tuberculin skin testing leads to desensitisation in naturally infected tuberculous cattle which is associated with elevated interleukin-10 and decreased interleukin-1 beta responses
}

\author{
Michael CoAd*, Derek Clifford, Shelley G. Rhodes, \\ R. Glyn Hewinson, H. Martin Vordermeier, Adam O. Whelan
}

TB Research Group, Veterinary Laboratories Agency, Woodham Lane, New Haw, Addlestone, Surrey KT15 3NB, United Kingdom

(Received 12 August 2009; accepted 16 October 2009)

\begin{abstract}
The principal surveillance tool used to control bovine tuberculosis in cattle is the removal of animals that provide a positive response to the tuberculin skin-test. In this study we performed a longitudinal investigation of the immunological and diagnostic consequences of repeated short-interval skin-tests in cattle naturally infected with Mycobacterium bovis. Tuberculin skin-test positive cattle were subjected to up to four further intradermal comparative cervical skin-tests at approximately 60-day intervals. A significant progressive reduction in the strength of the skin-test was observed after successive tests. In contrast, the magnitude of interferon- $\gamma$ (IFN- $\gamma$ ) responses was not influenced by repeat skin-testing either transiently around the time of each skin-test or longitudinally following repeated tests. A significant boost in blood interleukin-10 (IL-10) production was observed within 3 days following each skin-test although the magnitude of this boosted response returned to lower levels by day 10 post-test. The application of a novel multiplex assay to simultaneously measure seven cytokines and chemokines also identified that skin-testing resulted in a significant and progressive reduction in antigen specific interleukin-1 $\beta$ (IL-1 $\beta$ ) whilst confirming stable IFN- $\gamma$ and elevated IL-10 responses in the blood. Therefore, we have demonstrated that in cattle naturally infected with $M$. bovis, repeat short-interval skin-testing can lead to a progressive reduction in skin-test responsiveness which has potential negative consequences for the detection of infected animals with marginal or inconclusive skin-test responses. The desensitising effect is associated with decreased IL-1 $\beta$ and elevated IL-10 responses, but importantly, does not influence antigen specific IFN- $\gamma$ responses.
\end{abstract}

bovine tuberculosis / skin-testing / gamma-interferon / interleukin-10 / interleukin-1ß

\section{INTRODUCTION}

Bovine tuberculosis (TB) is a zoonotic disease caused by the bacterial pathogen Mycobacterium bovis. In developed countries where control and eradication of bovine TB continues to be a problem, the significance of its

\footnotetext{
* Corresponding author:m.coad@vla.defra.gsi.gov.uk, this article is subject to British Crown copyright.
}

impact on the farming community and government control agencies is primarily economic, although the zoonotic potential of bovine TB remains an important consideration. Furthermore, in developing countries where little or no control policies are in place, the disease may be a more significant public health concern $[3,17,38]$.

The principal diagnostic surveillance tool used to control bovine TB is the tuberculin skin-test, with removal of animals found to be 
positive (reactors). The test involves the intradermal injection of tuberculin, a purified protein derivative (PPD) prepared from a culture of M. bovis or M. avium. The two most commonly used test formats are the caudal fold test (CFT) and the single intradermal comparative cervical tuberculin (SICCT) test (reviewed by [24]). The CFT is a single intradermal test in which a bovine tuberculin PPD (PPD-B) is injected into the caudal fold at the base of the tail. The SICCT involves the intradermal injection of PPD-B and avian PPD (PPD-A) at separate sites in the skin of the neck. For both tests, skin induration reactions are read $72 \mathrm{~h}$ following tuberculin administration, this being a classic manifestation of a cell mediated delayed-type hypersensitivity (DTH) response characterised in cattle by the predominant infiltration of $\mathrm{CD}^{+}$and $\mathrm{CD}^{+}$ T-cells and macrophages [8]. The SICCT is the more specific test format since the comparative use of PPD-A allows for the measurement of non-specific sensitisation to environmental mycobacteria which can cross-react with antigens present in PPD-B [33]. For this reason, in the UK the SICCT is used in bovine TB surveillance operations.

Since the tuberculin skin-test is an in vivo test requiring administration of mycobacterial derived antigens, it has the potential to influence subsequent immuno-diagnostic tests. Indeed, the reduction in the ability of a $M$. bovis infected animal to respond for a period of time following subcutaneous administration of a tuberculin was reported as early as 1891 [23]. More recent studies have demonstrated that if a SICCT test is performed 7 days following either a CFT or SICCT test, significant skin-test desensitisation in reactor cattle can occur $[14,28]$. There have also been several studies in recent years investigating the influence of the skin-test on interferon- $\gamma$ (IFN- $\gamma$ ) responses in blood since measurement of IFN- $\gamma$ responses are being increasingly used as an adjunct to the skin-test to improve disease diagnosis $[5,30]$. For example, in cattle experimentally infected or sensitised with M. bovis, a boost in the PPD-B stimulated IFN- $\gamma$ response was observed 3 days following a CFT [27, 37] whereas in naturally infected cattle, we and others have shown that the SICCT test did not influence IFN- $\gamma$ response around the time of the test $[2,7,12]$.

Previous investigations on the influence of skin-testing on subsequent diagnostic tests have focused on either its effect on a single repeat skin-test within a period of 3 to 59 days or, in the case of its influence on IFN- $\gamma$ responses, the period 3-10 days immediately after a skin-test has been investigated. Yet, on farms where bovine TB is identified or suspected, it is common for multiple short-interval SICCT tests to be performed at intervals no shorter than 60 days since multiple tests may be required before all infected cattle are identified and/or to allow resolution of the status of animals providing "inconclusive" test results.

In the current study we performed a longitudinal investigation of the immunological and diagnostic consequences of repeated short-interval skin-tests in cattle naturally infected with $M$. bovis. We investigated the influence of repeat tests on longitudinal changes in subsequent skintests and also on cytokine and chemokine responses in antigen stimulated blood cultures. Our results provide novel insights into the immune parameters influenced by skin-testing which are discussed in the context of their practical relevance to the diagnosis of bovine TB.

\section{MATERIALS AND METHODS}

\subsection{Animals}

To investigate the effect of repeated short-interval skin-test responses, tuberculin skin-test reactor cattle $(n=23)$ were recruited from nine UK farms with a confirmed history of bovine TB (convenience sampling dependent on availability) and housed in a secure segregated bio-containment facility at the Veterinary Laboratories Agency (VLA, Weybridge, UK). Under standard field surveillance procedures, these animals would normally have been sent for slaughter on the basis of their diagnosis for bovine TB. The study group consisted of 13 males and 10 females, of 8 different breeds and with an age range of 5-26 months at recruitment (mean 14 months). Blood samples were taken from the animals at regular time points during the study period including immediately before and after the skin-tests performed at VLA. At the end of the holding period, 
these reactors were subjected to a detailed postmortem examination and severity of the pathology was scored according to a semi-quantitative system as previously described [35]. Tissue samples were homogenised and plated onto modified 7H11 Agar plates [11] and incubated at $37{ }^{\circ} \mathrm{C}$ for 6 weeks. All 23 reactors were visibly lesioned and/or culture positive for M. bovis. Isolates were found to be of spoligotypes SB0140, SB0263 and SB0274 which correspond to the three types most commonly found in mainland GB [32].

To investigate intracellular cytokine responses, a further 12 tuberculin skin-test reactor cattle were sourced from farms with a confirmed history of bovine TB. Confirmation of disease status by post mortem examination was not performed for these animals since they were retained for ongoing studies. TB negative Holstein-Fresian cattle $(n=19)$ were sourced as calves from TB-free farms and housed in separate accommodation from the reactors. All cattle experiments were cleared by local ethical review and animals procedures performed in accordance with British Home Office requirements.

\subsection{Tuberculin skin-tests}

The SICCT test was performed and interpreted according to European Communities Commission Regulation (Number 1226/2002 amending Annexes $\mathrm{A}$ and $\mathrm{B}$ of the consolidated Council Directive 64/432/EEC) using PPD-A and PPD-B (VLA). The disclosing SICCT skin-test (D) was performed by Animal Health veterinary officers. Following animal recruitment, up to four further SICCT skin-tests, referred to as ST1-ST4, were performed by the same veterinary officer at VLA, with an interval of approximately 60 days between tests. At ST1, 8 of 23 reactor animals received a CFT in accordance with the Bovine Tuberculosis Eradication Uniform Methods and Rules effective 22 January 1999 of the US Animal and Plant Health Inspection Service (APHIS), the remaining 15 received the SICCT test. All 23 animals received SICCT tests at ST2 and ST3. Thirteen of these cattle were retained for a 4 th SICCT test (ST4). The 19 TB-negative animals, and the 12 naturally infected "reactors" used to investigate intracellular cytokine response, each received a single SICCT test.

Under the severe interpretation of the SICCT test, animals are deemed reactors if the animal shows a positive (1 $\mathrm{mm}$ or greater) PPD-B reaction and a negative (less than $1 \mathrm{~mm}$ ) PPD-A reaction or the PPD-B reaction exceeds the PPD-A reaction by more than $2 \mathrm{~mm}$. An inconclusive result is indicated where an animal shows positive PPD-A and PPD-B reactions and the PPD-B reaction is 1 or $2 \mathrm{~mm}$ less, equal to, or 1 or $2 \mathrm{~mm}$ more than the PPD-A reaction.

\subsection{Measurement of cytokines in whole blood cultures}

Heparinised whole blood cultures were stimulated with either PPD-A (10 $\mu \mathrm{g} / \mathrm{mL}$, VLA), PPD-B $(10 \mu \mathrm{g} / \mathrm{mL}$, VLA), staphylococcal enterotoxin B $(1 \mu \mathrm{g} / \mathrm{mL}$ Sigma-Aldrich, St. Louis, MO, USA) or no-antigen control. Cultures were set up within $8 \mathrm{~h}$ of blood collection and cultured at $37{ }^{\circ} \mathrm{C} / 5 \% \mathrm{CO}_{2}$.

\subsubsection{IFN- $\gamma$ whole blood ELISA}

Antigen stimulated IFN- $\gamma$ was measured in $24 \mathrm{~h}$ whole blood culture supernatants using the commercially available BOVIGAM ${ }^{\circledR}$ ELISA (Prionics AG, Zurich, Switzerland). Results are expressed as the background-corrected optical density measured at $450 \mathrm{~nm}\left(\mathrm{OD}_{450 \mathrm{~nm}}\right)$ with the PPD-A response subtracted from the PPD-B response.

\subsubsection{Interleukin-10 (IL-10) whole blood ELISA}

IL-10 levels were measured in $72 \mathrm{~h}$ whole blood supernatants using a modification of methodology previously described [20]. This modified assay used the luminescence based detection methodology of Meso-Scale Discovery (MSD), Gaithersburg, MD, USA. In summary, MSD MA6000 96-well plates were coated overnight at $4{ }^{\circ} \mathrm{C}$ with mouse antibovine IL-10 (clone CC318, AbD-Serotec, Oxford, UK) diluted in phosphate buffered saline (PBS, Invitrogen, Paisley, UK). Plates were washed between each step using PBS containing $0.05 \%$ Tween 20 (Sigma-Aldrich) and subsequent incubations were performed at room temperature. Following a $1 \mathrm{~h}$ blocking step using PBS $/ 0.05 \%$ Tween 20 with $4 \%$ Bovine Serum Albumin (BSA, Sigma-Aldrich), duplicate samples were incubated for $1 \mathrm{~h}$ and recombinant IL-10 calibration standards (IAH, Compton, UK) were included on each plate. Plates were washed, and then biotinylated mouse anti-bovine IL-10 (clone CC320, AbD-Serotec) diluted in dilution buffer (PBS/Tween20 with 1\% BSA) was added for $1 \mathrm{~h}$. Following a further wash, Streptavidin-Sulphotag (MSD product R32AD-1, $0.3 \mu \mathrm{g}$ / $\mathrm{mL}$ ) was added for $1 \mathrm{~h}$. Finally, plates were washed and coated with MSD Buffer-T (MSD Product R92TC-1) and read on the MSD-6000 Sector Imager. Results are expressed as biological units per $\mathrm{mL}$ (U, as previously defined [20]). 


\subsubsection{IFN- $\gamma, I L-1 \beta, I L-4, I L-10, I L-12, M I P-1 \beta$ and $T N F-\alpha$ multiplex MSD assay}

Simultaneous detection of IFN- $\gamma$, IL-1 $\beta$, IL-4, IL-10, IL-12, MIP-1 $\beta$ and TNF- $\alpha$ was performed in $72 \mathrm{~h}$ whole blood culture supernatants using a custom bovine 7-plex cytokine/chemokine assay developed in collaboration with MSD. Multiplex 96-well plates were supplied with each of the target capture antibodies (commercially available: Bovine IFN- $\gamma$ (Mabtech, Stockholm, Sweden); IL-4 and TNF- $\alpha$ (Endogen, Rockford, IL, USA); IL-10 and IL-12 (AbD-Serotec); IL-1 $\beta$ and human cross-reactive MIP-1 $\beta$ (MSD)) pre-spotted onto seven spatially separated locations in each well. Incubations were performed at room temperature. Plates were blocked with MSD assay buffer prior to addition of sample or standards. The standards were serially diluted in MSD dilution buffer with top concentration of standard shown in parenthesis: IFN- $\gamma$ (Endogen, $100 \mathrm{ng} / \mathrm{mL}$ ); IL-1 $\beta$ (Bovine IL-1 $\beta$ calibrator (MSD) $20 \mathrm{ng} / \mathrm{mL}$ ); IL-4 (Bovine IL-4 calibrator (MSD), $2 \mathrm{ng} / \mathrm{mL}$ ); IL-10 (IAH, $30 \mathrm{U} / \mathrm{mL}$ ); IL-12 (IAH, $1000 \mathrm{U} / \mathrm{mL}$ ) and MIP-1 $\beta$ (Human MIP-1 $\beta$ calibrator (MSD), $10 \mathrm{ng} / \mathrm{mL}$ ). Following a $2 \mathrm{~h}$ incubation, plates were washed and then incubated for a further $2 \mathrm{~h}$ with a combined cocktail of biotinylated secondary detection antibodies (IFN- $\gamma$, IL-1 $\beta$, IL-4, IL-10, IL-12, MIP-1 $\beta$ (all from MSD) and TNF- $\alpha$ (Endogen, PBOTNF-AB1)) and Streptavidin-Sulfotag (MSD) in MSD dilution buffer. Minimum detectable levels are as follows: IFN- $\gamma 0.046 \mathrm{ng} / \mathrm{mL}$; IL- $1 \beta$ $0.043 \mathrm{ng} / \mathrm{mL}$; IL-4 $3.19 \mathrm{pg} / \mathrm{mL}$, IL-10 $0.018 \mathrm{U} / \mathrm{mL}$, IL-12 $1.83 \mathrm{U} / \mathrm{mL}$ and MIP-1 $\beta 1.32 \mathrm{pg} / \mathrm{mL}$. After a final wash, plates were coated with MSD Buffer-T and luminescence signal measured on the MSD6000 . TNF- $\alpha$ responses are reported as luminescence light units due to the lack of a reliable standard whilst all other parameters are reported in calibrated units extrapolated from standard curves.

\subsection{Intracellular flow cytometric cell staining (ICS)}

Whole blood was collected into tubes containing sodium heparin and $0.5 \mathrm{~mL}$ aliquots were diluted 1:1 in RPMI-1640 containing 10\% FBS, non-essential amino acids, $100 \mathrm{U} / \mathrm{mL}$ penicillin, $100 \mu \mathrm{g} / \mathrm{mL}$ streptomycin, $5 \times 10^{-5} \mathrm{M}$ 2-mercaptoethanol. These cultures were incubated in the presence of either 10 $\mu \mathrm{g} / \mathrm{mL}$ PPD-B (VLA), $10 \mu \mathrm{g} / \mathrm{mL}$ pokeweed mitogen (PWM) (Sigma-Aldrich) or media alone for $6 \mathrm{~h}$ at $37{ }^{\circ} \mathrm{C} / 5 \% \mathrm{CO}_{2}$ followed by a further $16 \mathrm{~h}$ in the presence of $10 \mu \mathrm{g} / \mathrm{mL}$ Brefeldin-A (Sigma-Aldrich). Red blood cells were lysed by 1:10 dilution of cultures in PharmLyse lysis buffer (BD Biosciences, San Diego, CA, USA) in accordance with manufacturers recommendations.

Simultaneous surface staining for the bovine cell markers CD4, CD8, CD14, and TcR $\delta$-chain was performed using the following antibody:fluorochrome conjugates; CD4:AlexaFluor647, clone CC8 (AbDSerotec); CD8:AlexaFluor700, clone CC63 (antibody from $\mathrm{AbD}-\mathrm{Serotec}$, custom conjugation by Invitrogen); CD14:PE-Cy5.5, cross reactive human clone TuK4 (Caltag Laboratories, Carlsbad, CA, USA); TcR- $\delta$ :Pacific Orange, clone GB21A (antibody from VMRD, Pullman, WA, USA, conjugation performed at VLA using an Invitrogen conjugation kit). Staining was performed in a $100 \mu \mathrm{L}$ volume for $15 \min$ at $4{ }^{\circ} \mathrm{C}$ in the presence of an amine reactive Violet dead-cell staining reagent (Invitrogen). Cells were washed with Hanks' Buffered Saline (Invitrogen) containing $2 \%$ fetal calf serum (Sigma-Aldrich), $0.05 \%$ Na-azide (Sigma-Aldrich) and then fixed in 1\% paraformaldehyde (BD Biosciences) overnight at $4{ }^{\circ} \mathrm{C}$.

Fixed cells were permeabilised for $20 \mathrm{~min}$ at $4{ }^{\circ} \mathrm{C}$ using a permeabilisation buffer containing Saponin (BD Biosciences). Permeabilised cells were washed and then co-stained for intracellular bovine IFN- $\gamma$ and IL-10 for $30 \mathrm{~min}$ at $4{ }^{\circ} \mathrm{C}$ using the following antibody conjugates; IFN- $\gamma: \mathrm{PE}$, clone CC302 (AbD-Serotec); IL10, clone CC318 (AbD-Serotec) pre-labelled with an IgG2b specific Zenon Alexafluoro 488 label (Invitrogen). Following a final two washes, analysis of labelled cells was performed using a Beckman Coulter CyanADP flow cytometer with Summit v4.3 analysis software. The seven-colour, nine-parameter gating strategy excluded doublet-cell populations and also any cells providing positive staining for the Violet dead-cell stain. The optimal working titre for all antibodies was determined prior to use and single colour controls were included to ensure application of optimal compensation settings. The mean number of cells analysed in the live-cell gate was 185114 (95\% CI, 177969-192259).

\subsection{Statistical analysis}

Significant changes in the magnitude of the skintest following repeated tests, or in the magnitude of cytokine/chemokine response at different sampling occasions in relation to the skin-test, were calculated using ANOVA, with application of Bonferroni Multiple Comparison post analysis test where significance $(p<0.05)$ was identified. Correlations of immune or pathological parameters were performed using the Spearman Rank correlation. These, and the regression 
analysis, were calculated using the Prism software program (Graphpad Inc., San Diego, CA, USA).

\section{RESULTS}

\subsection{Influence of repeat short-interval skin-tests}

The SICCT skin-test response was measured at up to four consecutive short-interval tests in cattle naturally infected with $M$. bovis. Regression analysis of the mean response at each test demonstrated a highly significant decay in the magnitude of the comparative response following successive tests $\left(p=0.0003, r^{2}=0.9925\right.$, Fig. 1). The difference in response between the disclosing and subsequent tests reached significance at ST3 and continued to be significantly different at ST4 $(p<0.01)$. The decay in the comparative response was a consequence of a significant decay in the PPD-B response $\left(p=0.0097, r^{2}=0.9206\right)$ but not in the PPDA response $\left(p=0.4501, r^{2}=0.2\right)$ (data not shown). Since it was not clear whether this skin-test desensitisation was directly due to the repeated skin-tests or an indirect consequence of disease progression during the holding period, we investigated the relationship between the drop in the skin-test response and disease severity in individual animals. No significant correlation was observed between the pathology score and the difference in the skintest response between disclosing and ST3 tests in individual animals $\left(p=0.577, r^{2}=0.020\right.$, data not shown).

\subsection{Influence of the skin-test on blood IFN- $\gamma$ production}

Production of IFN- $\gamma$ prior to, and 3 and 10 days following 3 SICCT tests was measured in antigen stimulated blood cultures using the BOVIGAM ELISA. Analysis of comparative PPD responses in those animals for which we had comparative SICCT pre-, day-3 and day-10 test data demonstrated no significant change in the magnitude of the response following any of the three skin-tests (Fig. 2A). In addition, the comparative PPD IFN- $\gamma$ response in all 23 study animals measured prior to each

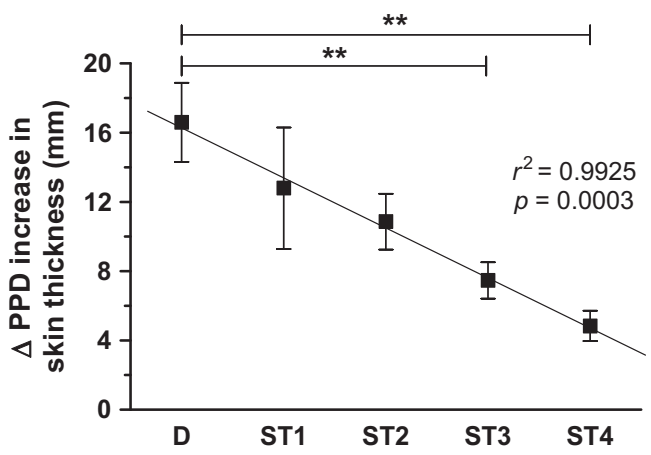

Figure 1. Influence of repeat SICCT testing on subsequent skin-tests. SICCT responses were measured in naturally infected cattle at approximately 60 day intervals $(n=23,15,23,23$ and 13 respectively). The comparative PPD response at each test is presented as the mean value for each group ( \pm standard error). The line represents the significant decreasing linear regression with $r^{2}$ and $p$ values indicated, and significant differences between $\mathrm{D}$ and subsequent tests is indicated by $* *(p<0.01)$.

of the three skin-tests demonstrated no significant difference between consecutive tests (data not shown). Therefore, repeat SICCT shortinterval application did not change the strength of the IFN- $\gamma$ response either transiently around the time of the skin-test or longitudinally following repeated tests.

Since it has been reported that in contrast to the SICCT [2, 7, 12] the CFT format of the skin-test can boost IFN- $\gamma$ response 3 days post-test [27, 37], we took the opportunity to perform a direct comparison of the effect of CFT and SICCT on IFN- $\gamma$ responses in a cohort of the cattle. At ST1 only, a CFT was performed in eight of the study animals. In those animals that received the CFT, a significant increase in the comparative PPD IFN- $\gamma$ response was observed 3 days after the test $(p<0.05)$ (Fig. 2B). The boost in the comparative response was a consequence of a significant increase in the PPD-B stimulated response $(p=0.0149)$ rather than a change in the PPDA response $(p=0.6488)$. By day 10 , responses had returned to a level comparable to pre-test responses. No difference in the IFN- $\gamma$ response was observed in the 15 cattle that received the 

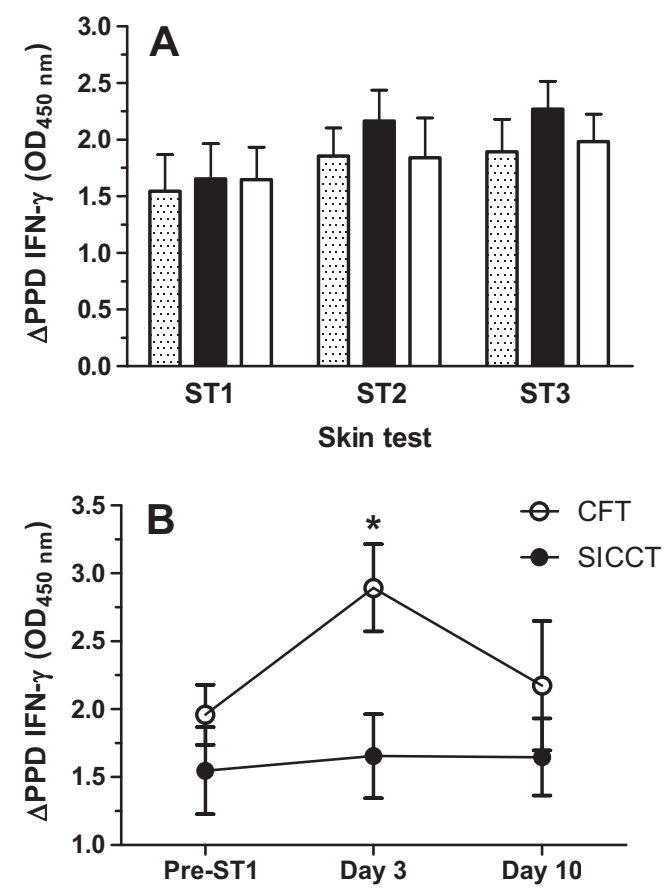

Figure 2. Influence of the skin-test on blood IFN- $\gamma$ responses. (A) IFN- $\gamma$ responses were measured in blood samples from cattle naturally infected with $M$. bovis around the time of three consecutive SICCT tests (ST1-ST3, $n=15,13,23$ respectively). Blood samples were tested prior to (shaded bars) and at 3 (solid bars) and 10-days (unshaded bars) following each of three skin-tests. (B) Comparison of the effect of CFT and SICCT tests on blood IFN- $\gamma$ responses was measured in 8 and 15 cattle respectively. Responses were measured prior to, and 3 and 10 days post skin-test (ST1) and the response in cattle given the CFT and SICCT tests are show as open and closed circles respectively. Where a response at either day 3 or 10 was significantly different from the pre-test response, this is indicated $\left({ }^{*} p<0.05\right)$. For both panels, data are presented as mean $\left(\mathrm{OD}_{450 \mathrm{~nm}}\right)$ of the PPD-B stimulated response minus the PPD-A response ( \pm standard error).

SICCT. For those 8 cattle that received the CFT at ST1, but then received SICCT tests at ST2 and ST3, no boost in the IFN- $\gamma$ response was observed following either of the subsequent SICCT tests (data not shown).

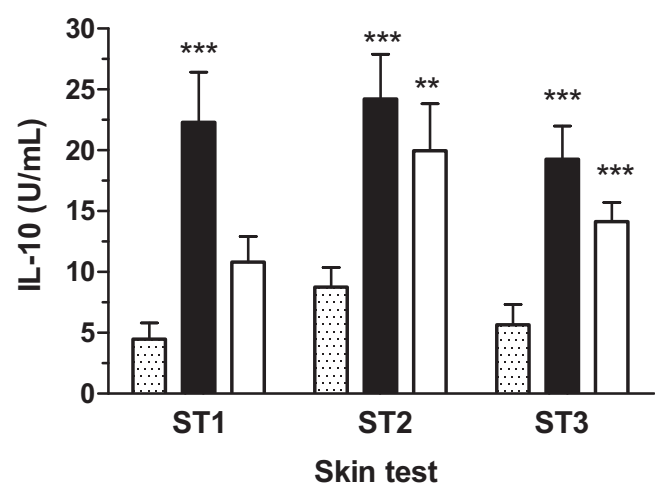

Figure 3. Influence of the SICCT on blood IL-10 responses. IL-10 responses were measured in blood samples from naturally infected cattle around the time of three consecutive SICCT tests (ST1-ST3). Blood samples were tested prior to (shaded bars) and at 3 (solid bars) and 10-days (unshaded bars) following each of three skin-tests $(n=15,10$ and 18 animals respectively). Data are presented as mean standardised IL-10 ELISA responses $(\mathrm{U} / \mathrm{mL})$ of PPD-B stimulated response minus the nil antigen response ( \pm standard error). Where a response at either day 3 or 10 was significantly different from the pre-test response, this is indicated by $* * p<0.01$ or $* * * p<0.001$.

\subsection{Influence of the skin-test on blood IL-10 production}

We next investigated if the SICCT skin-test exerted an influence on the induction of the immune regulatory cytokine IL-10. Production of IL-10 prior to, and 3 and 10 days following, 3 SICCT tests was measured in PPD-B stimulated blood cultures using a luminescence based ELISA assay (Fig. 3). For all three SICCT tests there was a significant increase in IL-10 responses 3 days post-test $(p<0.01)$, which remained elevated at day 10 . Comparison of the 3 respective pre-test responses demonstrated an increase in the magnitude of the IL-10 response, particularly with regards to ST1 and ST2, but this trend was not quite significant (Fig. 3, $p=0.0562$ ).

To evaluate the specificity of the transient boost in PPD-B stimulated IL-10 responses, blood culture supernatants were also tested 
prior to, and following, a SICCT in 19 TB-free cattle. All 19 cattle were SICTT negative (data not shown). No or only marginal PPD-B specific IL-10 could be detected in these animals following their skin-test (mean response in 23 naturally infected cattle $=5.2 \mathrm{ng} / \mathrm{mL}$, 95\% CI 3.9-6.4; mean response in 19 TB-free cattle $=0.7 \mathrm{ng} / \mathrm{mL}, 95 \%$ CI $0.4-0.9$ ). Furthermore, no significant change in PPD-B induced IL-10 pre and post skin-test was observed in these animals $(p=0.178$, data not shown).

\subsection{Intracellular expression of IFN- $\gamma$ and IL-10 by bovine $T$-cells}

Since we had shown that transient skin-test boosting of IL-10 was antigen specific, we investigated whether T-cells could be the source of IL-10. We measured intracellular expression of IL-10 and IFN- $\gamma$ from CD4 ${ }^{+}, \mathrm{CD}^{+}$and $\gamma \delta^{+}$ T-cells isolated from blood from 12 naturally infected cattle 3 days after a SICCT test (Fig. 4). Despite the observation that PWM could induce expression of IL-10 in a population of $\mathrm{CD}^{+}$cells, we were unable to detect any PPD-B induced expression of IL-10 from any of these T-cell populations (Fig. 4B). In contrast, ICS staining for IFN- $\gamma$ confirmed that $\mathrm{CD}^{+}$T-cells were the primary source of PPDB stimulated IFN- $\gamma$ (Fig. 4A). The ICS protocol also allowed staining for $\mathrm{CD} 14^{+}$cells which constituted $2.2 \%(0.4-4.0,95 \% \mathrm{CI})$ of total live cells analysed. We were unable to detect any antigen induced expression of IL-10 or IFN- $\gamma$ in this non-adherent $\mathrm{CD}_{1} 4^{+}$population (data not shown).

\subsection{Measurement of IFN- $\gamma$, IL-10, IL-1 $\beta$, IL-4, IL-12, TNF- $\alpha$ and MIP-1 $\beta$ by MSD multiplex assay}

To investigate the effect of repeat skin-testing on a broader range of cytokines including IFN- $\gamma$, IL-10, IL-1 $\beta$, IL-4, IL-12, TNF- $\alpha$ and the chemokine MIP-1 $\beta$, we used a novel luminescence based multiplex assay (Fig. 5). This assay allowed simultaneous measurement of these seven immune parameters in the same sample. PPD-B stimulated responses were measured in blood collected before skin-tests ST1,
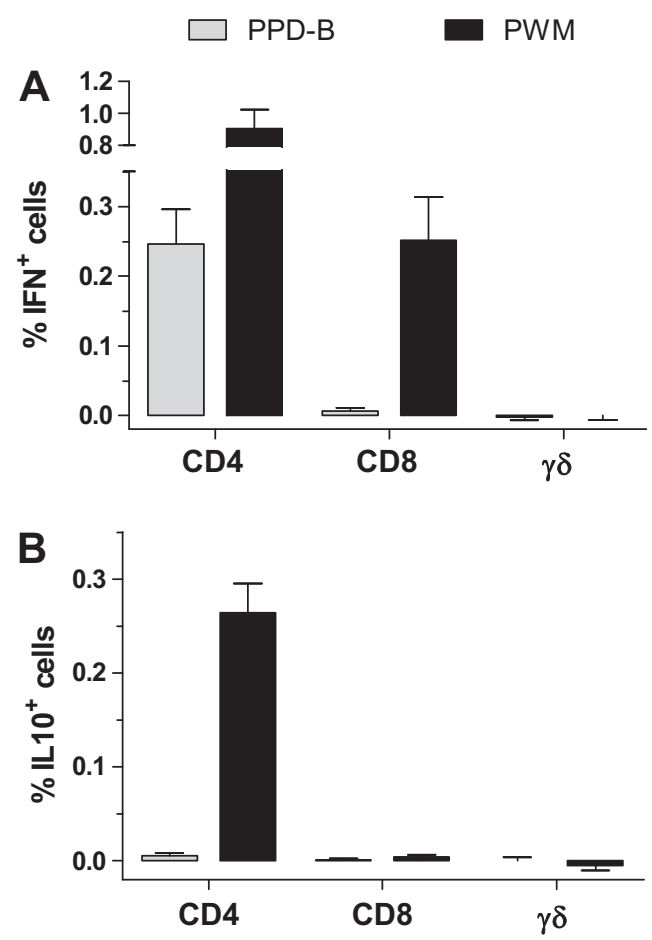

Figure 4. Intracellular IFN- $\gamma$ and IL-10 expression by bovine T-cell subsets. Blood from tuberculin reactor cattle $(n=12)$ was collected 3 -days post skin-test. ICS expression of IFN- $\gamma$ and IL-10 by CD4, CD8 and TcR- $\delta$ T-cell subsets was determined using lysed whole blood cultures stimulated with either PPD-B or PWM. The percentage of $\mathrm{CD}^{+}, \mathrm{CD}^{+}{ }^{+}$and $\mathrm{TcR}-\delta^{+}$cells expressing IFN- $\gamma$ or IL-10 are shown in panels (A) and (B) respectively. PPD-B induced responses (no-antigen response subtracted) as represented by grey bars and PWM responses black bars (mean \pm standard error).

ST2 and ST3. Confirming the previous BOVIGAM ELISA data (Fig. 2), IFN- $\gamma$ responses remained unaffected by repeat skin-testing. No significant modulation in IL-4 (data not shown), IL-12, TNF- $\alpha$ or MIP-1 $\beta$ was observed following repeat skin-testing. Interestingly, a significant increase in the strength of IL-10 responses was observed when comparing the magnitude of the response between ST1 and ST2 and ST3 $(p<0.05)$, supporting the observation that we described in Section 3.3. In contrast, IL-1 $\beta$ responses progressively decreased 



Figure 5. Measurement of IFN- $\gamma$, IL-10, IL-12, IL-1, TNF- $\alpha$ and MIP-1 $\beta$ using a multiplex luminescence assay. Blood was collected from cattle naturally infected with M. bovis $(n=23)$ immediately prior to each of three successive skin-tests (ST1-ST3). PPD-B stimulated responses in whole blood cultures are presented at each time-point as the mean value ( \pm standard error) with the no-antigen control response subtracted. TNF- $\alpha$ data is presented in units of luminescence whilst all other responses are calibrated against a recombinant standard that was run on each assay plate. Significance between ST1 and subsequent readings is indicated by $*(p<0.05)$.

and were significantly lower at ST3 compared with ST1 $(p<0.05)$. The decrease in the IL-1 $\beta$ response between in ST1 and ST3 for individual animals did not correlate with their pathology scores $\left(p=0.144, r^{2}=0.09884\right)$.

The multiplex assay was also used to measure PPD-B stimulated responses in supernatants from bloods taken prior to, and 3 and 10 days post-ST1 $(n=23)$. A significant increase in IL-10 expression 3-days after testing was observed which was consistent with the single-plex IL-10 ELISA results presented in Figure 3 . No significant change in the magnitude of any of the other parameters was observed (data not shown).

\section{DISCUSSION}

We investigated the effect of repeat shortinterval SICCT skin-tests in UK cattle naturally infected with $M$. bovis and observed a progressive and significant decrease in reaction sizes at successive tests. Possible explanations for this include either that the skin-test itself exerted a desensitising effect or that during the study period, disease progression resulted in reduced cell mediated responses. The latter explanation must be considered since T-cell anergy has been reported in cattle [21]. However, we observed no correlation between the severity of pathological disease and their respective decrease in skin-test responses. Furthermore, had disease progression been the reason for decreased cellular responsiveness, and thus reduced DTH reactions, we would also have expected to see a decrease in IFN- $\gamma$ responses which was not the case. Therefore, it seems more likely that despite applying the recommended 60 day retest interval, repeat SICCT testing led to increasing desensitisation at subsequent tests.

The reduction in the ability of $M$. bovis infected animals to respond for a short period of time following administration of a tuberculin is well recognised $[14,18,28]$. When naturally infected cattle were re-tested after 60 days in an 
earlier study, the response was not significantly different from that measured in the initial test yet the authors noted that even after 60 days, complete return to "original sensitivity" did not occur in all animals. We also did not detect a significant decrease in the strength of skin-test response following the first, or even second, repeat skin-test. Not until the third test did the difference between the repeat and original test became significant. Furthermore, since we were able to undertake up to four repeat skin-tests, we could demonstrate that the progressive decrease in skin-test responsiveness was highly significant. A recent study in experimentally infected cattle demonstrated that responses to a SICCT test performed 15 weeks postchallenge were significantly reduced compared with those observed at 8 weeks earlier [34]. The authors speculated that the drop in skin-test responsiveness was a consequence of the previous test which is consistent with our data in naturally infected cattle.

Our model in naturally infected cattle suggests that the period of desensitisation between tests is longer than previously thought and that successive short-interval skin-tests will result in progressive desensitisation. Our data could therefore be of practical importance when considering the diagnosis of infected animals with "inconclusive" skin-test responses. In such cases, animals are usually serially re-tested 60 days later to clarify their status. Given that skin-test responses may still be depressed 60 days following the previous test, this raises the possibility that a weak or indeterminate response could give rise to a negative test outcome. Therefore, the possibility of repeat testing resulting in false-negative test outcomes in infected cattle with indeterminate test responses cannot be excluded.

Despite the observation that repeat SICCT testing resulted in desensitisation to subsequent skin-tests, blood IFN- $\gamma$ responses were not affected. We and others have previously shown that a SICCT does not exert a significant effect on PPD stimulated IFN- $\gamma$ responses measured in blood from naturally infected cattle collected 3-10 days after the test $[2,7,12]$. The data from our current study extends these findings to demonstrate that the magnitude of PPD stimulated IFN- $\gamma$ in blood also remains unaffected follow- ing repeated SICCT tests. In contrast, it has been reported that the CFT can boost IFN- $\gamma$ response immediately following the test $[27,37]$ which has led to some debate to concile the discrepancy with results from previously studies where the SICCT has been applied $[2,7,12]$. We therefore took the opportunity to perform a direct comparison of the effect of the SICCT and CFT tests on IFN- $\gamma$ responses in some of our study animals. The observation that the boost in the IFN- $\gamma$ response 3 days after the test was only observed in those animals that recieved the CFT clearly demonstrates that the CFT and SICCT skin-tests can exert different effects on peripheral blood responses thereby explaining the apparent discrepancies in the literature. Whilst it would be interesting to investigate the basis for these differences, this was outside the scope of the study.

IL-10 is an important cytokine which can regulate macrophage dependant in vitro IFN- $\gamma$ production from Th1 cells $[4,9,25]$ and can also inhibit in vivo DTH responses in mice $[22,31]$. In infected cattle, the SICCT has been found to boost production of IL-10 in PPD-B stimulated blood cultures [6]. In the current study, we have confirmed and extended these findings. We observed a highly significant increase in PPD-B stimulated blood IL-10 responses within 3 days of each skin-test which remained elevated at day 10 . Although the peak magnitude of the IL-10 boost was largely transient the IL-10 ELISA showed a trend for stronger IL-10 responses immediately prior to ST2 compared with ST1 which was confirmed as significant when measured using the multiplex assay. Therefore, in addition to the strong skin-test induced transient boost in IL-10, there was also evidence of a longitudinal increase in IL-10 between tests. Given that IL-10 can inhibit DTH responses [22, 31], this may provide an explanation for the well recognised shortterm desensitising effect of the tuberculin skin-test, whilst also contributing to the longitudinal desensitisation observed in the current study. Future in vivo studies will be required to investigate this further.

We did not observe a skin-test induced boost in PPD-B stimulated IL-10 responses in naive uninfected cattle. Furthermore, in the naturally 
infected cattle, mitogen stimulated IL-10 responses were not affected by the skin-test (data not shown). This suggested an antigen specific mechanism involving cells of the acquired immune response. In humans and mice, a population of $\mathrm{CD}^{+} \mathrm{CD} 25^{+}$T-cells expressing FoxP3 has most commonly been associated with T-regulatory properties [1, 10, 29]. A recent study in cattle found no regulatory properties associated with the equivalent bovine cell population [13]. Instead, the authors reported regulatory properties from a subset of $\gamma \delta$ T-cells and expression of IL-10 was largely detected from $\mathrm{CD} 4^{+}$monocytes. We were unable to identify the source of IL-10 expression in our model although our data suggests that it was not produced by $\mathrm{CD}^{+}{ }^{+}$T-cells which were however the primary source of IFN- $\gamma$ expression. It is possible that adherent monocytes might be the source of the IL-10 since adherent cultured cells would not have been analysed by the protocol used and would therefore warrant future investigation.

In humans with $\mathrm{TB}$, the IFN- $\gamma / \mathrm{IL}-10$ ratio has been used to define disease severity [15]. An inverse relationship was found between the two cytokines with IL-10 being elevated in patients with more extensive disease. In experimentally infected cattle, elevated IL-10 has also been associated with increased pathology [36] whilst in vitro neutralisation of bovine IL-10 in blood cultures can upregulate IFN- $\gamma$ expression [6]. Our results in naturally infected cattle suggest that the relationship between IFN- $\gamma$ and IL-10 expression in bovine blood cultures is not as clear cut. For example, we observed significant boosting of antigen specific IL-10 responses after skin-testing in the absence of changes in the IFN- $\gamma$ response. We also found no correlation between the IFN- $\gamma /$ IL-10 ratio and extent of pathology (data not shown). Therefore, in contrast to the data from human TB cases, IL-10 is a less reliable bio-marker for disease progression in naturally infected cattle.

The use of a novel bovine multiplex assay to simultaneously measure seven immune parameters proved to be a valuable technique to further explore bovine immune responses. The multiplex IFN- $\gamma$ and IL-10 responses were consistent with data from the single cytokine ELISA assays thereby validating this novel methodology and also providing confirmation of our previously discussed data. Additionally, we were able to identify that antigen induced IL-1 $\beta$ responses decreased progressively following repeat skin-testing. In mice IL- $1 \beta$ is required for antigen specific T-cell activation and induction of a DTH response [26] and IL-1 has a role in the suppression of PPD induced DTH during BCG infection [19]. It is therefore interesting that the longitudinal decrease in antigen induced IL-1 $\beta$ appears to associate with longitudinal skin-test desensitisation in our cattle. This is despite the response of other pro-inflammatory associated cytokines such as IFN- $\gamma$, TNF- $\alpha$ and IL-12 remaining unaffected. Studies in human macrophages have shown that increased IL-10 inhibits IL-17 induced production of IL-1 $\beta$ [16]. Given that in vivo administration of PPD can lead to elevated IL-10 responses, it would be tempting to speculate that the resulting skin-test desensitisation involves a regulatory mechanism involving IL-10 and IL-1 $\beta$. Further studies will be required to determine their in vivo role in the bovine DTH reaction.

In summary, we have demonstrated that repeat short-interval application of the tuberculin skin-tests can lead to longitudinal skin-test desensitisation whilst not influencing IFN- $\gamma$ blood based diagnosis of bovine TB. This skin-test desensitisation was found to be associated with antigen-specific elevated levels of IL-10 and decreased IL- $1 \beta$ in blood suggesting a possible regulatory role for these cytokines in the development of tuberculin skin-test responses. Improved understanding of mechanisms involved in skin-test responses will not only be of benefit in the diagnosis of bovine TB but also has practical relevance to the application of skin-testing as a diagnostic tool in the clinical setting.

Acknowledgements. We would like to acknowledge the VLA Animal Services Unit staff for animal husbandry support, Animal Health officers for recruitment of cattle and Dr Jayne Hope, Institute of Animal Health, Compton, UK for recombinant bovine IL-10 and IL-12 standards. This work was funded by the Department for the Environment, Food and Rural Affairs (DEFRA), UK. 


\section{REFERENCES}

[1] Belkaid Y., Role of Foxp3-positive regulatory T cells during infection, Eur. J. Immunol. (2008) 38:918-21.

[2] Coad M., Hewinson R.G., Clifford D., Vordermeier H.M., Whelan A.O., Influence of skin testing and blood storage on interferon- $\gamma$ production in cattle affected naturally with Mycobacterium bovis, Vet. Rec. (2007) 160:660-662.

[3] Cosivi O., Grange J.M., Daborn C.J., Raviglione M.C., Fujiura T., Cousins D., et al., Zoonotic tuberculosis due to Mycobacterium bovis in developing countries, Emerg. Infect. Dis. (1998) 4:59-70.

[4] Couper K.N., Blount D.G., Riley E.M., IL-10: the master regulator of immunity to infection, J. Immunol. (2008) 180:5771-5777.

[5] De La Rua-Domenech R., Goodchild A.T., Vordermeier H.M., Hewinson R.G., Christiansen K.H., Clifton-Hadley R.S., Ante mortem diagnosis of tuberculosis in cattle: a review of the tuberculin tests, $\gamma$-interferon assay and other ancillary diagnostic techniques, Res. Vet. Sci. (2006) 81:190-210.

[6] Denis M., Wedlock D.N., McCarthy A.R., Parlane N.A., Cockle P.J., Vordermeier H.M., et al., Enhancement of the sensitivity of the whole-blood gamma interferon assay for diagnosis of Mycobacterium bovis infections in cattle, Clin. Vaccine Immunol. (2007) 14:1483-1489.

[7] Doherty M.L., Monaghan M.L., Bassett H.F., Quinn P.J., Effect of a recent injection of purified protein derivative on diagnostic tests for tuberculosis in cattle infected with Mycobacterium bovis, Res. Vet. Sci. (1995) 58:217-221.

[8] Doherty M.L., Bassett H.F., Quinn P.J., Davis W.C., Kelly A.P., Monaghan M.L., A sequential study of the bovine tuberculin reaction, Immunology (1996) 87:9-14.

[9] Fiorentino D.F., Bond M.W., Mosmann T.R., Two types of mouse T helper cell. IV. Th2 clones secrete a factor that inhibits cytokine production by Th1 clones, J. Exp. Med. (1989) 170:2081-2095.

[10] Fontenot J.D., Gavin M.A., Rudensky A.Y., Foxp3 programs the development and function of CD4+CD25+ regulatory T cells, Nat. Immunol. (2003) 4:330-336.

[11] Gallagher J., Horwill D.M., A selective oleic acid albumin agar medium for the cultivation of Mycobacterium bovis, J. Hyg. (1977) 79:155-160.

[12] Gormley E., Doyle M.B., McGill K., Costello E., Good M., Collins J.D., The effect of the tuberculin test and the consequences of a delay in blood culture on the sensitivity of a gamma-interferon assay for the detection of Mycobacterium bovis infection in cattle, Vet. Immunol. Immunopathol. (2004) 102:413-420.

[13] Hoek A., RuttenV.P., Kool J., Arkesteijn G.J., Bouwstra R.J., Van Rhijn I., Koets A.P., Subpopulations of bovine $\mathrm{WC}^{+} \gamma \delta \mathrm{T}$ cells rather than $\mathrm{CD} 4^{+} \mathrm{CD} 25^{\text {high }}$
Foxp $^{+} \mathrm{T}$ cells act as immune regulatory cells ex vivo, Vet. Res. (2009) 40:06.

[14] Hoyle F.P., Suppression of tuberculin skin reactivity in cattle: a case report, Surveillance (1990) 17:28.

[15] Jamil B., Shahid F., Hasan Z., Nasir N., Razzaki T., Dawood G., Hussain R., Interferon $\gamma /$ IL 10 ratio defined the disease severity in pulmonary and extra pulmonary tuberculosis, Tuberculosis (2007) 87:279-287.

[16] Jovanovic D.V., Di Battista J.A., Martel-Pelletier J., Jolicoeur F.C., He Y., Zhang M., et al., IL-17 Stimulates the production and expression of proinflammatory cytokines, IL-1 $\beta$ and TNF- $\alpha$, by human macrophages, J. Immunol. (1998) 160:3513-3521.

[17] Kazwala R.R., Daborn C.J., Sharp J.M., Kambarage D.M., Jiwa S.F.H., Mbembati N.A., Isolation of Mycobacterium bovis from human cases of cervical adenitis in Tanzania: a cause for concern?, Int. J. Tuberc. Lung Dis. (2001) 5:87-91.

[18] Kerr W.R., Lamont H.G., McGirr J.L., Studies on tuberculin sensitivity in the bovine, Vet. Rec. (1946) 58:443-446, 451-454.

[19] Kobayashi K., Allred C., Cohen S., Yoshida T., Role of interleukin 1 in experimental pulmonary granuloma in mice, J. Immunol. (1985) 134:358-364.

[20] Kwong L.S., Hope J.C., Thom M.L., Sopp P., Duggan S., Bembridge G.P., Howard C.J., Development of an ELISA for bovine IL-10, Vet. Immunol. Immunopathol. (2002) 85:213-223.

[21] Lepper A.W.D., Pearson C.W., Corner L.A., Anergy to tuberculin in beef cattle, Aust. Vet. J. (1977) 53:214 216.

[22] Li L., Elliot J.F., Mosmann T.R., IL-10 inhibits cytokine production, vascular leakage, and swelling during T Helper 1 cell-induced delayed-type hypersensitivity, J. Immunol. (1994) 153:3967-3978.

[23] Miller E.B., Tuberculous cattle problem in the United States to 1917, Hist. Med. Vet. (1989) 14:1-64.

[24] Monaghan M.L., Doherty M.L., Collins J.D., Kazda J.F., Quinn P.J., The tuberculin test, Vet. Microbiol. (1994) 40:111-124.

[25] Moore K.W., De Waal Malefyt R., Coffman R.L., O'Garra A., Interleukin-10 and the Interleukin-10 receptor, Annu. Rev. Immunol. (2001) 19:683-765.

[26] Nambu A., Nakae S., Iwakura Y., IL-1 $\beta$, but not IL$1 \alpha$, is required for antigen-specific $\mathrm{T}$ cell activation and induction of local inflammation in the delayed-type hypersensitivity responses, Int. Immunol. (2006) 18:701-712.

[27] Palmer M.V., Waters W.R., Thacker T.C., Greenwald R., Esfandiari J., Lyaschenko K.P., Effects of different tuberculin skin-testing regimens on Gamma Interferon and antibody responses in cattle experimentally 
infected with Mycobacterium bovis, Clin. Vaccine Immunol. (2006) 13:387-394.

[28] Radunz B.L., Lepper A.W.D., Suppression of skin reactivity to bovine tuberculin in repeat tests, Aust. Vet. J. (1985) 62:191-194.

[29] Roncarolo M., Gregori S., The validity of Foxp3 to define human and mouse regulatory $\mathrm{T}$ cells, Eur. J. Immunol. (2008) 38:901-937.

[30] Rothel J.S., Jones S.L., Corner L.A., Cox J.C., Wood P.R., The gamma-interferon assay for diagnosis of bovine tuberculosis in cattle: conditions affecting the production of gamma-interferon in whole blood culture, Aust. Vet. J. (1990) 69:1-4.

[31] Schwarz A., Grabbe S., Riemann H., Aragane Y., Simon M., Manon S., et al., In vivo effects of interleukin10 on contact hypersensitivity and delayed-type hypersensitivity reactions, J. Invest. Dermatol. (1994) 103:211-216.

[32] Smith N.H., Gordon S.V., De La Rua-Domenech R., Clifton-Hadley R.S., Hewinson R.G., Bottlenecks and broomsticks: the molecular evolution of Mycobacterium bovis, Nat. Rev. Microbiol. (2006) 4:670-681.

[33] Stenius R., Differentiation by tuberculin testing of infection in cattle due to the human, bovine and avian types of tubercle bacilli, Vet. Rec. (1938) 50:633-637.
[34] Thom M.L., Morgan J.H., Hope J.C., VillarrealRamos B., Martin M., Howard C.J., The effect of repeated tuberculin skin testing of cattle on immune responses and disease following experimental infection with Mycobacterium bovis, Vet. Immunol. Immunopathol. (2004) 102:399-412.

[35] Vordermeier H.M., Chambers M.A., Cockle P.J., Whelan A.O., Simmons J., Hewinson R.G., Correlation of ESAT-6 specific gamma interferon production with pathology in cattle following Mycobacterium bovis BCG vaccination against experimental bovine tuberculosis, Infect. Immun. (2002) 70:3026-3032.

[36] Welsh M.D, Cunningham R.T., Corbett D.M., Girvin R.M., McNair J., Skuce R.A., et al., Influence of pathological progression on the balance between cellular and humoral immune responses in bovine tuberculosis, Immunology (2005) 114:101-111.

[37] Whipple D.L., Palmer M.V., Slaughter R.E., Jones S.L., Comparison of purified protein derivatives and the effect of skin testing on results of a commercial gamma interferon assay for diagnosis of tuberculosis in cattle, J. Vet. Diagn. Invest. (2001) 13:117-122.

[38] Zinsstag J., Kazwala R.R., Cadmus I., Ayanwale L., Mycobacterium bovis in Africa, in: Thoen C.O. (Ed.), Mycobacterium bovis infection in animals and humans, Blackwell Publishing, USA, 2006, pp. 199-210. 